\title{
Wie Erinnertes lebendig wird. Tote und Touristen in Hans Chlumbergs Wunder um Verdun
}

Comment la remémoration prend vie. Morts et touristes dans Wunder um Verdun/Miracle autour de Verdun de Hans Chlumberg

How things remembered become alive. The Dead and the Tourists in Hans

Chlumberg's Wunder um Verdun/Miracle around Verdun

\section{Christa Karpenstein-Eßbach}

\section{OpenEdition Journals}

Édition électronique

URL : http://journals.openedition.org/ceg/2091

DOI : 10.4000/ceg.2091

ISSN : 2605-8359

Éditeur

Presses Universitaires de Provence

\section{Édition imprimée}

Date de publication : 15 juin 2014

Pagination : 197-205

ISSN : 0751-4239

Référence électronique

Christa Karpenstein-Eßbach, «Wie Erinnertes lebendig wird. Tote und Touristen in Hans Chlumbergs Wunder um Verdun », Cahiers d'Études Germaniques [Online], 66 | 2014, Online erschienen am: 17 Dezember 2017, abgerufen am 20 November 2020. URL : http://journals.openedition.org/ceg/2091 DOI : https://doi.org/10.4000/ceg.2091 


\title{
Wie Erinnertes lebendig wird. Tote und Touristen in Hans Chlumbergs Wunder um Verdun
}

\author{
Christa KARPENSTEIN-EßBACH
}

Universität Mannheim

I.

Erinnern, die Pflege des Gedächtnisses, das Gedenken gehören zu den Aufgaben, an die wir immer wieder erinnert werden - so auch zum 100. Jahrestag des katastrophischen Beginns des 20. Jahrhunderts. Aber auch jenseits der Daten von Großereignissen haben Gedächtnis und Erinnerung eine prominente Stellung im Gebiet der menschlichen Fähigkeiten - ob es sich nun um die grassierende Angst vor der Krankheit des Gedächtnisverlustes, vor der Auslagerung des Gedächtnisses in technische Apparate oder um die vor amoralischer Vergeßlichkeit handelt. Mnemosyne hat Lethe nicht besiegt, wohl aber in eine untergeordnete Stellung verwiesen. Die starke Position dieser Muse verdankt sich den an Erinnerung und Gedächtnis geknüpften Erwartungen, denn sie sollen zum Besseren beitragen, eine heilsame Funktion für die Zukunft haben oder die Wiederholung eines erinnerten, meist katastrophischen Ereignisses verhindern. Schließlich hat das griechische Wort aletheia, dessen kleine Vorsilbe Lethe negiert, dafür gesorgt, daß die Wahrheit selbst damit zu tun hat, daß wir nicht vergessen. ${ }^{1}$

Hans Chlumberg hat mit seinem 1932 erschienenen Theaterstück Wunder um Verdun einen der herausragenden Erinnerungsorte an den Ersten Weltkrieg gewählt. Verdun ist der Ort für politisch ausgerichtete Gedenktage. Zum 50. Jahrestag der Schlacht wurde auf Veranlassung von Charles de Gaulle eine rein französische Feier mit großem Aufwand ausgerichtet ${ }^{2} ; 1984$ war Verdun die Kulisse der Begegnung von Mitterand und Kohl mit dem symbolischen Höhepunkt der deutsch-französischen Versöhnungsgeste des gemeinsamen Händehaltens. Aber nicht nur dies: Verdun gehört zu den S. 15.

S. Harald WeInRICH, Lethe. Kunst und Kritik des Vergessens, München, Beck, 2005, 23.

\footnotetext{
S. Josef MÜLLER-MAREIN, „Die Veteranen von Verdun“, in Die Zeit vom 3.6.1966, Nr.
} 
bevorzugten Adressen eines Kriegsgräbertourismus, der schon in der Zwischenkriegszeit beginnt. Dem heutigen Reisenden wird unter den Sehenswürdigkeiten des Ortes genannt: die rote Zone, das Beinhaus von Douaumont, Fort Douaumont mit seinen Bunkeranlagen und Fort Vaux. Die französische Polizei kontrolliert, daß von den Touristen keine Kriegsgegenstände oder Knochen mitgenommen werden. Der Verband Connaissance de la Meuse richtet jedes Jahr die Event-Aufführung Des Flammes à la Lumière / Von den Flammen zum Licht über die Schlacht von Verdun aus, mit, wie das Tourismusbüro informiert, 300 Schauspielern, 900 Kostümen, 1000 Scheinwerfen, $40 \mathrm{~km}$ Kabel sowie vielen Spezialeffekten. Geworben wird mit der Aufforderung: „Tauchen Sie einen Abend in die Geschichte ein. Erleben Sie die Schlacht von Verdun in einer atemberaubend realistischen Freilichtaufführung“, die „emotionale Momente“ verspricht. ${ }^{3}$ Weit jenseits der Lebenszeit derer, die die Gräber ihrer gefallenen Männer, Väter und Söhne besuchen, sind Stätten wie Verdun Besucheradressen geworden und geblieben. ${ }^{4}$

Dabei verdankt sich die herausragende Stellung Verduns einer langen Reihe von politischen Ereignissen, Konflikten und Serien institutionell gestützter Erinnerungen, die zum Bestand eines deutsch-französischen Gedächtnisses gehören. Vom Zweiten Weltkrieg aus gesehen, legen sich sechs Daten mit ihren jeweils künftigen Erinnerungspotentialen übereinander. 1940 findet nach 1916 die zweite Schlacht um Verdun statt. 1916 erinnert man sich an 1870/71 und den langen Widerstand Verduns, das nach dem Ende des deutsch-französischen Krieges französisch bleibt, weshalb die Eroberung Verduns nach 1870 eine Planspielaufgabe für Generalstabsoffiziere wird. 1648 war Verdun, das 1552 vom französischen König Heinrich II. annektiert worden war, mit dem Westfälischen Frieden an Frankreich gefallen - beides wiederum Daten, an die im Ersten Weltkrieg erinnert wird. Im Vertrag von Verdun 843 wird das von Karl dem Großen gegründete Fränkische Reich aufgeteilt, und es kommt zur Embryonalbildung von Deutschland und Frankreich. Nimmt man neben 843 als ein zweites Eckdatum die Versöhnungsgeste zwischen Mitterand und Kohl im Jahre 1984, so handelt es sich bei Verdun um einen Ort, an den eine mehr als tausendjährige deutsch-französische Erinnerungsgeschichte adressiert wird. ${ }^{5}$

Chlumberg verlegt die Spielzeit seines Stückes von 1930 in die Zukunft, in den August 1939 mit den 25-Jahr-Feiern zum Beginn des Ersten Weltkrieges. $\mathrm{Da} \beta$ es sich um das um einen Monat differierende Datum des Beginns des Zweiten Weltkrieges handelt, konnte Chlumberg nicht mehr

\footnotetext{
${ }^{3}$ www.tourisme-lorraine.fr

${ }^{4}$ Der Volksbund Deutsche Kriegsgräberfürsorge e.V. gibt jährlich einen Kalender heraus, der, einem Tourismuskatalog vergleichbar, mit Bildern von schönen Landschaften kombinierte Fotos von den dort liegenden Kriegsgräberstätten zeigt und für „Reisen mit uns“ wirbt.

5 S. die umfangreiche Untersuchung von Matti MÜNCH, Verdun. Mythos und Alltag einer Schlacht, München, Martin Meidenbauer Verlagsbuchhandlung, 2006. Speziell zum „Schlachtfeldtourismus“ s. S. $460 \mathrm{ff}$.
} 
wissen, denn er ist bei einer Probe seines Stückes in den Orchestergraben gestürzt und 1930 vor der Uraufführung in Leipzig an den Folgen des Unfalls gestorben. Wunder um Verdun hatte großen, auch internationalen Erfolg, wurde aber wegen seines „,faulen Pazifismus“ angegriffen und später von den Nationalsozialisten verboten. ${ }^{6} \mathrm{Da} \beta$ es sich um ein, gewiß unkonventionelles, pazifistisches Stück handelt, wird man schnell feststellen können. Hier interessieren zwei andere Fragen: die nach der Dynamik von Erinnern und Gedenken, und die nach den kulturellen Differenzen zwischen den Vertretern der internationalen Personnage der Gedenkfeierlichkeiten.

II.

Wunder um Verdun umfaßt dreizehn Szenen, Bilder genannt, die zu Episoden verknüpft werden. Es geht nicht um die Vergegenwärtigung kriegerischer Realität im theatralen Jetzt, sondern um die Erinnerung an den Krieg, deren theatrale Gegenwärtigkeit in die Zukunft projiziert wird. Da das Stück unter Literaturwissenschaftlern nahezu unbekannt ist, ist es notwendig, dieses eine Werk genauer vorzustellen, hier ohne Beschreibungen des Kontextes. ${ }^{7}$ Das Stück beginnt mit dem Besuch der Schlachtfelder von Verdun durch eine internationale Touristengruppe - „Vergnügungsreisende“ genannt -, führt über die je nationalen Kriegsgedenkfeiern in Frankreich, Deutschland und England, die Auferstehung der toten Soldaten mit deren Besuchen in ihrer Heimat und ihrem Auftritt auf einer internationalen Konferenz bis zur Rückkehr der Toten in ihre Gräber. Neben einigen aus dem großen Heer der Toten werden insgesamt etwa sechzig Personen namentlich benannt, darunter vor allem Touristen und Politiker. Das touristische Interesse wird im Gedenkinteresse der vielen politischen Akteure und der Angehörigen der Gefallenen gespiegelt, denn die Regieanweisung weist darauf hin, daß die Personen der ersten und letzten Szene mit den Auftritten der Touristen und

${ }^{6}$ Elisabeth PABLE, „Der vergessene Welterfolg: Hans von Chlumberg“, in Literatur und Kritik, H. 36/37, Salzburg, Otto Müller, 1969, S. 382-395. Zur zeitgenössischen Rezeption: Arno SCHIROKAUER, „Die Partei der 13 Millionen“, in Das Tagebuch, 11. Jg., 2. Halbjahr, Berlin, Tagebuchverlag, 1930, S. 1724 f. Alfred POLGAR, „Wunder um Verdun“, in Die Weltbühne, 28. Jg. 2. Halbjahr, Berlin, Verlag der Weltbühne, 1932, S. 397 f. Fritz BIEBER, „Hans Chlumberg: Wunder um Verdun“, in Die literarische Welt, 8. Jg., Nr. 38, Berlin, 16. Sept. 1932, S. 7. Bieber fragt sich, „was würde wohl Chlumberg zu der neuen Fassung seines Stückes im Deutschen Theater sagen“, und bemerkt: „Oh - wie vorsichtig ist es geworden, wie taktlos taktvoll, wie wunderbar beruhigend - alle fremden Staatsmänner dreschen chauvinistische Phrasen, nur der deutsche Kanzler spricht schlicht und treuherzig ergriffen." $P$. K. G. (Patrick Geoghegan?), „The Play Miracle at Verdun. By Hans Chlumberg. At the Comedy Theatre London”, in New Blackfriars, Jg. 14, Oxford (Blackwell Publishing), 1933, S. $74 \mathrm{f}$. Hilde SPIEL berichtet von einer Lesung des Stückes durch Attila Hörbiger in der Österreichischen Gesellschaft für Literatur im Jahre 1967, in Theater 1967. Chronik und Bilanz eines Bühnenjahres, Velbert, Erhard Friedrich Verlag, 1968, S. 29.

$\mathrm{Zu}$ Chlumbergs Drama im Kontext s. Christa KARPENSTEIN-EßBACH, „Krieg und Geschichte. Zur literarischen Repräsentation des Ersten Weltkriegs im Ausgang der Weimarer Republik“, in Elisabeth Guilhamon, Daniel MeYer (Hrsg.), Die streitbare Clio. Zur Repräsentation von Macht und Geschichte in der Literatur, Frankfurt, Berlin, Peter Lang, 2010, S. 109-126. 
die Personen in den Bildern zwei bis zwölf von denselben Schauspielern dargestellt werden sollen. Die Toten hingegen treten in je einzelnen Schauspielerkörpern auf, womit sich das Stück zugleich gegen Gedenkkonzepte des Unbekannten Soldaten wendet. Dem Stück vorangestellt sind Verse aus Hesekiel 37, 1 und 4-10, in denen davon berichtet wird, wie das Totenfeld, in diesem Fall Israels, durch den aus dem Mund des Propheten strömenden Odem Gottes lebendig wird. Aber dies alte Wunder der Auferstehung hat in Chlumbergs Stück jedoch keine erlösende Kraft mehr, vielmehr werden die wiederkehrenden Toten des Krieges zum Medium, in dem sich die fatale Dynamik eines verlebendigenden Erinnerns entfaltet.

Die Übertragung dieses alten Gedankens der Auferstehung auf die Toten des Weltkrieges ist auch in zwei Versionen des Films J'accuse von Abel Gance aus den Jahren 1918 und 1938 zu finden. Es scheint sich um eine Phantasie zu handeln, die in der Luft liegt. Wir wissen nicht, ob Chlumberg den Film von 1918 gesehen hat. Aber ein vergleichender Blick auf beide Filme macht den Unterschied in der Bedeutung, die diese Auferstehung hat, deutlich. Beide Filme von Gance mit ihrem jeweils melodramatischen Charakter enden mit der Schlußszene der Auferstehung. Gance meinte in Hinblick auf den ersten Film, dass die Wiederkehr der Toten aus dem Film ein transzendierendes Epos der Gefühle macht. ${ }^{8}$ Diese Toten sind Opfer, und ihr Opfer soll einen Sinn erhalten; insofern darf man in dem Film die Artikulation eines moralischen Protestes sehen, die die Lebenden in der Anerkennung des Opfers mit den Toten versöhnt. Der zweite Film endet ebenfalls mit der Auferstehung der Toten und ihrer Rückkehr in die Heimat. Die Anweisung im Script lautet: „In this last scene ... do about ten highly emotional flashes between a woman of 40 , daughter of 20 , son of 20 , grandchildren who embrace their rediscovered dead and who swear never more to hate or to fight. With German, English, Russians, Poles, Italians, Belgians, Americans, in all their respective languages, do: 1) a war widow 2) daughter of a dead soldier 3) son of a dead soldier 4) mother of a dead soldier“. In beiden Fällen finden die Toten ihre Aufnahme, so daß „the sacrifice of the last war rescues the present.“" ${ }^{\star 9}$ Chlumbergs Stück hingegen setzt in diese Form der Rettung der Gegenwart durch die Treue gegenüber den Toten weniger Hoffnung und mißtraut offenbar der präventiven Kraft des Kriegsgedenkens und den friedensstiftenden Folgen, die ihm zugeschrieben werden. Daß drei Jahre vor dem nächsten Krieg sich zehntausend Kriegsveteranen aus verschiedenen Ländern in Fort Douaumont treffen und schwören, den Frieden zu verteidigen, konnte Chlumberg allerdings auch nicht wissen.

\footnotetext{
${ }^{8}$ Gance „,considered that the return of the dead made the film a transcendent epic of emotion“, zitiert nach John HORNE, „Film and Cultural Demobilisation after the Great War: The Two Versions of J'accuse by Abel Gance (1918 and 1938)“, in Hanna Diamond, Simon KITSON (Hrsg.), Vichy,Resistance, Liberation. New Perspectives on Wartime France, Oxford, New York, Berg, 2005, S. 131-141, hier S. 134.

${ }^{9}$ Ibid., S. 138
} 
Am Beginn von Wunder um Verdun steht eine groteske Situation, wenn statt Trauernder oder andächtig Gedenkender die Touristengruppe auftritt, in der sogleich ein Streit u. a. über das angemessene Verhältnis zwischen Eintrittspreis und der Anzahl der Toten ausbricht. Auf die Preiskritik von Amerikanern und Engländern antwortet der Besucher aus Frankreich: „Wer zum Henker, hat Sie denn herübergerufen? Wären Sie doch zu Hause geblieben und hätten sich Ihre eigenen Schlachtfelder angesehen, wenn Ihnen die unseren nicht passen!“ “10, worauf sich der Streit darüber fortsetzt, welchem Land mit wie vielen seiner Toten die Ehre des Sieges zukommt. Eine gewisse Beruhigung aller setzt in dem Moment ein, in dem ihnen ein Massengrab gezeigt wird, in dem Deutsche und Franzosen gemeinsam liegen. Diese Eröffnungsszene hat ihre historische Resonanz im nach 1918 einsetzenden Kriegsgräbertourismus, den schon Karl Kraus attackierte, ${ }^{11}$ aber sie hat so wenig dokumentarischen Charakter wie die folgenden Szenen, die als dramatische Analyse der Folgen von Erinnern und Gedenken zu interpretieren sind.

Die Feierlichkeiten in Paris und Berlin, die anschließend zu sehen sind, beginnen mit Aufrufen zum Gedenken an die Gefallenen, verwandeln sich aber sogleich in konfliktbeladene Konfrontationen. Im Falle Frankreichs habe das Opfer der Toten „den Feind aufs Knie gezwungen“, im Falle Deutschlands dürfe es „nicht vergebens bleiben“ - jeweils verbunden mit Ausrufen: es lebe Frankreich respektive Deutschland. Wie der französische Ministerpräsident phantasiert, daß „ein Wunder geschähe“ und die Toten, „ekstatisch von uns empfangen“, unter die Lebenden träten, um diesen zu versichern: „Übergegangen ist unser Geist auf euch!“, so bekennt der deutsche Reichskanzler, der Geist der Toten „hat sich von euren Gräbern erhoben. Er kam zu uns, er ging auf uns über ${ }^{12}$ “. Chlumbergs Stück nimmt diese die Toten verlebendigende Phantasie literarisch ernst, wenn die Toten von einem Boten, einem Engel mit der Hoffnung erweckt werden, daß die Auferstehung „Ströme von solcher Glückseligkeit über dieses Gottesvolk auf Erden ausbrechen und aufquellen (lassen werde), daß unser aller $\mathrm{Ha} ß$ unterwühlt und unterwaschen würde.“13 Der Bote hebt an dieser Stelle die heilsame Kraft hervor, die dem Erinnern zugesprochen wird. Angesichts der realen Präsenz der Toten, die innenpolitisch Unruhe stiftet, sehen sich die Weltkriegsnationen zur Einigkeit genötigt, die im gemeinsamen Abweis der Toten besteht. Sie sind in die Nachkriegsgesellschaften nicht integrierbar. Alle Regierungen rühmen sich, mit Heldenfriedhöfen, Denkmälern oder der „internationalen Sehenswürdigkeit des Grabmals des Unbekannten Soldaten“

\footnotetext{
${ }^{10}$ Hans ChlumBerg, Wunder um Verdun, Berlin, S. Fischer, 1932, S. 15.

${ }^{11}$ Karl KRAUS druckt in Die Fackel Nr. 577-582, November 1921 ein Inserat der Basler Nachrichten, das für solche Fahrten wirbt, zusammen mit seinem Kommentar „Reklamefahrten zur Hölle“. Edlef Köppens Roman Heeresbericht von 1930 (Kronberg/Ts. 1976, S. 309) gibt das Inserat eines Berliner Reisebüros wieder.

${ }^{12}$ Hans ChlumBerg, Wunder um Verdun, S. 26, 29.

${ }^{13} \mathrm{Ibid}$, S. 32.
} 
für die Toten getan zu haben, „was man nur tun konnte“, und fordern sie auf : „kehret darum zurück, Unselige [...] Denn - ausgestoßen sollt ihr sein und exkommuniziert.“14 Damit konnten die Toten nach einem zweifachen post mortem zur Einigungsinstanz einer neuen politischen Gegenwart werden.

Aber das Stück beläßt es nicht bei dieser Lösung. Ausgerechnet die von den Toten aufgeworfene Friedensfrage provoziert den gegenseitigen Verdacht mangelnder Friedenswilligkeit unter den Politikern aller Seiten. Dabei kommt es zu einer neuen, aber gegenüber der ersten anders konturierten Gemeinsamkeit unter den Nationen. Die erste war über die Gedenkpraxen gestiftet worden, wonach „,der Tod den Nationen und Armeen (gehört) ${ }^{15}$ “. Die zweite besteht in der Einigkeit über die dauerhafte und immer zu erneuernde Funktion des Objekts des Gedenkens. Unter Zustimmung aller Teilnehmer der internationalen Konferenz erläutert Professor Dr. Steppach, ein wissenschaftlicher Sachverständiger, dessen Nationalität nicht genannt wird :

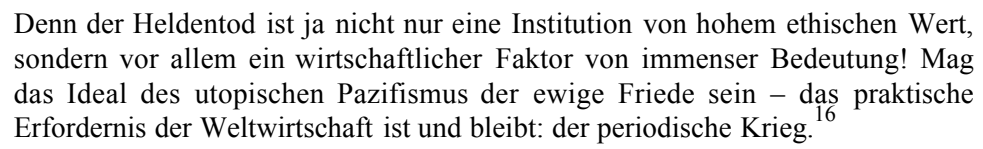

$\mathrm{Zu}$ dem ethisch begründeten Wert des Heldentodes tritt dessen funktionale Legitimation hinzu.

Chlumbergs Stück verschränkt zwei Konfliktlinien miteinander: den transnationalen Antagonismus von Lebenden und Toten, und den der nationalen Polarisierung. Die Dynamik beider wird als ein dramatischer Prozeß vorgeführt, in dem Friedhöfe und Gedenkfeiern zu Orten werden, an denen das kollektive Gedächtnis als Kriegstreiber entsteht. An einer zentralen Stelle des Dramas reflektiert der Bote die Modalitäten des Erinnerns in den Veranstaltungen des Gedenkens. Während die Lebenden „sonst ihre Toten meist in viel kürzerer Zeit (vergessen)“", gilt für diese Toten eine andere Logik.

Ihr aber, Tote des großen Krieges, seid unvergessen. Nicht verblaßt ist euer Andenken und nicht friedlich verklärt, wie das anderer, die gestorben sind. Nein. Weiß glüht es und wühlt Tag um Tag die Lebenden von neuem auf. Es macht, daß sie euer nicht mit Resignation gedenken, sondern mit all ihrer Leidenschaft! ${ }^{17}$

Die Hoffnung des Boten auf Ströme von Glückseligkeit erfüllt sich nicht, und die Toten selbst wissen am Ende nicht mehr, warum sie zurückgekehrt

${ }^{4}$ Ibid., S. 106, 115.

${ }^{15}$ Ibid., S. 111.

16 Ibid., S. 112.

${ }^{17}$ Ibid., S. 31. S. zur Frage des Erinnerns auch: Brian MURDOCH, „Memory and prophecy among the war-graves: Hans Chlumberg's drama, Miracle at Verdun", in William KIDD, Brian MuRDOCH (Hrsg.), Memory and Memorials. The Commemorative Century, London, Ashgate, 2004, S. 92-104. 
waren. Man darf das Stück als Plädoyer dafür lesen, an die Stelle leidenschaftlichen Gedenkens ein Andenken zu setzen, das „,verblaßt“, weil andernfalls, mit den Worten des Propheten Hesekiel, „ein großes, sehr großes Heer" sich erheben könnte, das, weil die Erinnerung lebendig gehalten wurde, im Jahre 1939 wieder bereit stand.

So sehr die Schlacht um Verdun während des Krieges von Erinnerungen an die mit dem Ort verbundenen historischen Tiefenstrukturen unterfüttert war, so sehr wurde Verdun erst mit den in den zwanziger Jahren einsetzenden institutionell gestützten Erinnerungen zum geradezu mythisierten Ereignis, von dem der Auftrag erging, dem Beispiel der Gefallenen nachzufolgen. „Verdun! [...] immer wieder - die Jahrhunderte haben es gelehrt - wird Waffengeklirr um ihre Mauern toben", schreibt die Besucherin von Verdun, Betty Schneider, 1935 in ihrem Reisebericht „Bei den Toten von Verdun“"18.

III.

In einer zweiten Schicht von Chlumbergs Stück geht es nicht um die zukünftigen Folgen vergegenwärtigter Erinnerung, sondern um den mit dem Gedenken aufbrechenden, weit zurückliegenden Erfahrungsgrund von Selbstund Fremdwahrnehmungen, die sich als Stereotype zur Geltung bringen. Mit Verdun hat Chlumberg zweifellos einen Ort aufgerufen, der sich, wie viele andere auch, zur Erzählung von „Schlachtenmythen“ eignet und an den konkurrierende Aktualisierungen historischer Kämpfe oder Vergewisserungen nationaler Gründungen und Aufbrüche adressiert werden können. ${ }^{19}$ Solche Schlachtenmythen eignen sich zur Wiedererzählung alter Ereignisse und zur Mobilisierung von Affekten auf dem Hintergrund alter kriegerischer Konfrontationen. In Chlumbergs Stück treffen aber keine Protagonisten aufeinander, deren Gegnerschaften einen solchen „schlachtenmythischen“ Hintergrund hätten. Ihre Unterschiede liegen weit unterhalb von Gebieten der großen Politik; sie aktualisieren auch keinen kämpferischen oder tragischen Heroismus. Man könnte sie als Mentalitäten im Gebiet des Alltäglichen, als Kleinigkeiten bezeichnen. Chlumberg stattet seine touristischen und politischen dramatis personae mit national-kulturell gefärbten Eigenschaften, Vorlieben und Verhaltensweisen auf eine ins Groteske reichende Weise aus. Ihre Funktion im Stück ist ambivalent, denn zum einen haben sie den Charakter je besonderer Skurrilitäten, zum anderen sind sie ein sich aus der Geschichte speisender Quellgrund für ihre politische Funktionalisierung in Freund-Feind-Verhältnissen.

Dazu eine erste kleine Serie von Beispielen. In den Szenen fünf, sechs und sieben wird dem französischen und dem englischen Ministerpräsidenten sowie dem deutschen Reichskanzler nachts die Nachricht von der Auferstehung der Toten telefonisch übermittelt. Das französische

\footnotetext{
${ }_{18}$ Zit. nach Matti MÜNCH, Verdun. Mythos und Alltag einer Schlacht, S. 463. Zu „Verdun“ in der Weimarer Republik ibid., S. 439-466, S. 525 f.

${ }_{19}$ S. Gerd KRUMEICH, Susanne BRANDT (Hrsg.), Schlachtenmythen. Ereignis - Erzählung - Erinnerung, Köln, Weimar, Wien, Böhlau, 2003.
} 
Staatsoberhaupt verbringt die Nacht mit seiner Geliebten, ein wortgewandter Disput zwischen beiden über Liebesleben und Politik und der telefonische Zwischenfall werden mit der Versicherung beendet, es handele sich um eine Intrige der Presse. In Berlin reagieren der Reichskanzler und seine Frau, die sich mit „Vatchen“ und „Muttchen“ ansprechen, auf den Anruf, indem sie auf den Instanzenweg und Zuständigkeiten verweisen. In London läßt sich der Ministerpräsident, nach einem Gespräch mit seinem Kammerdiener über die demokratische Einrichtung des Telefons und den Verfall der aristokratischen Sitten, die seinem Diener mitgeteilte Nachricht weitersagen und bezeichnet sie als eine Angelegenheit des Kontinents. Deutsche Ordnung, englischer Snobismus, französische Erotik sind ein Potpourri aus Fremd- und Selbstzuschreibungen, die historisch latent vorhanden sind, aber aktualisiert werden und ebenso wie die Toten wiederauferstehen können.

Diese andere Auferstehung vollzieht sich, so führt das Stück vor, mit der Transformation erinnerter kultureller Unterschiede in Konfliktverstärker. Dazu die zweite kleine Serie. Die französische Touristin referiert auf die den Französinnen zugeschriebene besondere Anziehungskraft, indem sie der Engländerin und der Amerikanerin erklärt: „Mit ihren Pfunden und Dollars verteuern Sie uns bloß unsere Kleider - und Ihnen stehen sie doch nicht!“. Den Vorwurf des Franzosen an die Amerikaner, diese hätten nur für ihr Geschäft geblutet, kontert der Amerikaner, sie hätten, wie von Frankreich erwartet, den Krieg zur „Rettung der Kultur“ geführt. Die sorgfältige Pflege des Friedhofs führt der Amerikaner wiederum nicht auf kulturelle, sondern auf wirtschaftliche Interessen zurück: „Eure Fremdenindustrie würde zurückgehen, und eure Handelsbilanz sähe dann noch schlechter aus! ‘20 Ein deutscher Tourist zeigt sich interessiert an den Namen der Gefallenen, deren Arbeiten die Wissenschaft befördert haben, und wird vom französischen Friedhofswärter auf die Namen der einfachen Leute hingewiesen. ${ }^{21}$ Die zweite Beispielreihe macht die Logik deutlich, nach der schon lange bestehende Wahrnehmungen national-kultureller Besonderheiten zur Waffe des Stereotyps werden. Dabei werden die Fremdzuschreibungen jeweils in Anspruch genommen, um sie gegen den anderen zu richten. Die durchaus grotesken Konflikte zwischen den Touristen auf dem Friedhof am Beginn des Stückes werden im weiteren Verlauf in den politisch-militärischen Antagonismen gespiegelt. Allein die lebendigen Toten bleiben dieser Logik entzogen.

Nimmt man das Stück abschließend in den Blick, so liegt es zunächst nahe, es zu historisieren und auf die besondere Erfahrungslage nach dem Ersten Weltkrieg zu beziehen. ${ }^{22}$ In einer Situation, in der der Sieg weiterhin gefeiert und die Niederlage nicht anerkannt wurde, persistiert die kriegerische Bereitschaft. Chlumberg hat eben diesen die Weltkriegsnationen umfassenden

20 Ibid., S. 15 ff.

21 Ibid., S. 21

22 In internationaler Perspektive aufschlußreich: Jost DüLFFER, Gerd KRUMEICH (Hrsg.), Der verlorene Frieden. Politik und Kriegskultur nach 1918, Essen, Klartext, 2002. 
Dispositionen von Kollektiven Gestalt gegeben, und insofern darf man darin ein zeitdiagnostisches Stück sehen. Auch Karl Kraus hatte mit seinem Monumentaldrama „Die letzten Tage der Menschheit“ die kriegerische Mentalität seiner Zeit auf die Bühne gebracht, und zweifellos eignet sich auch das Theater auf besondere Weise dazu, die Verfaßtheit und die Dynamik von Kollektiven zur Darstellung zu bringen, während die Lyrik und die Prosa der Perspektive eines Einzelnen verhaftet bleiben müssen. ${ }^{23}$ Im Unterschied zu Kraus bewegt sich Chlumbergs Stück jedoch nicht in der Aktualität des Kriegsgeschehens selbst. ${ }^{24}$ Es handelt sich um die Projektion ins Zukünftige, die von der Vergangenheit genährt wird, und es ist der Geist der Erinnerung und des Gedenkens, der den dramatischen Konflikt aufs Neue hervortreibt.. Damit gewinnt das Drama eine über seine Historisierung im Kontext der unmittelbaren Nachkriegszeit hinaus allgemeine Dimension. Bis heute wird täglich ersichtlich, daß, um die Worte des Boten zu wiederholen, der Kriegstoten nicht mit Resignation, sondern mit Leidenschaft gedacht wird. Die von dem deutschen Touristen, einem Überlebenden von Verdun, mit seinen Zweifeln an den Gedenkfeiern formulierte Frage „Was will man denn für die da unten?" 25 bleibt bis zu den heutigen Kriegen so akut wie das Problem, ob Lethe hier der Mnemosyne an die Seite treten dürfte oder sollte.

\footnotetext{
${ }^{23}$ Zur Bedeutung von Gattungen für die literarischen Darstellungsweisen von Kriegen s. Christa KARPENSTEIN-ESSBACH, Orte der Grausamkeit. Die Neuen Kriege in der Literatur, München, Fink, 2011.

${ }^{24}$ Zum Vergleich von Kraus' und Chlumbergs Dramen s. P. J. BRANSCOMBE, „Some Depictions of the First World War in Austrian Drama“", in B. O. MURDOCH, M. G. WARD (Hrsg.), Studies in Modern Austrian Literature, Glasgow, University Printing, 1981, S. 74-86.

${ }^{25}$ Hans Chlumberg, Wunder um Verdun, S. 23.
} 